\section{Integração entre vigilância sanitária e assistência à saúde da mulher: um estudo sobre a integralidade no SUS}

\author{
Integration of health surveillance and women's \\ health care: a study on comprehensiveness \\ in the Unified National Health System
}

\author{
${ }^{1}$ Faculdade de Ciências \\ da Saúde, Universidade de \\ Brasília, Brasília, Brasil. \\ 2 Programa de Pós-graduação \\ em Saúde Coletiva, Fiocruz \\ Brasília, Brasília, Brasil. \\ 3 Assessoria de Planejamento, \\ Agência Nacional de \\ Vigilância Sanitária, \\ Brasília, Brasil. \\ ${ }^{4}$ Câmara dos Deputados, \\ Brasília, Brasil. \\ Correspondência \\ C. Maia \\ Programa de Pós-graduação \\ em Ciências da Saúde, \\ Faculdade de Ciências da \\ Saúde, Universidade de \\ Brasília. \\ SHIN CA 5, Lote G, Ed. Portal \\ do Lago Norte, apto. 506, \\ Brasília, $D F$ \\ 71503-505, Brasil. \\ csmaia2@gmail.com
}

\begin{abstract}
Comprehensiveness is a key principle in Brazil's Unified National Health System (SUS), approached from various perspectives, including linkage between services. The debate on this principle appears in health care, especially in the area of Women's Health, and in Health Surveillance guidelines. Since both areas target quality of health services, the aim of this study is to analyze the integration between Health Surveillance and Women's Health Care. This is a qualitative case study that interviewed Health Surveillance staff in health services and coordinators of Women's Health services. The findings point to the isolation of Health Surveillance within the health secretariats. The importance of integrating the two areas is cited by Women's Health administrators, but it is difficult to implement. Collaborative relations only occur in emergency situations. The Health Surveillance professionals believe that this lack of integration is due to the fact that women's health issues do not require their participation, and that at any rate they collaborate with the area through health inspections and talks. The study detected difficulties in achieving integration between the two areas, with the persistent challenge of linking health actions, especially with Health Surveillance.
\end{abstract}

Women's Health; Health Public Policy; Comprehensive Health Care; Health Surveillance
Christiane Maia 1,2,3

Dirce Guilhem 1

Geraldo Lucchese 2,4

\section{Integralidade: reflexos e reflexões}

A necessidade de se garantir serviços de saúde integrais surgiu com a ampliação das políticas sociais, marcada nas democracias ocidentais pela criação do National Health Service (NHS), no Reino Unido, o qual estabeleceu, em 1948, como princípio, a responsabilidade coletiva por " $\mathrm{com}$ prehensive health services" 1.

O Sistema Único de Saúde (SUS), conforme a Lei $n^{o} .8 .080^{2}$, reforçou esse ideal para o Brasil ao definir como um de seus objetivos garantir " $a$ assistência às pessoas por intermédio de ações de promoção, proteção e recuperação da saúde, com a realização integrada das ações assistenciais e das atividades preventivas". Essa mesma lei assume a integralidade da assistência como um princípio e a descreve como "conjunto articulado e contínuo das ações e serviços preventivos e curativos, individuais e coletivos, exigidos para cada caso em todos os níveis de complexidade do sistema" 2.

O Pacto em Defesa do SUS, dimensão integrante do Pacto pela Saúde, assume como compromisso para os gestores do sistema a consolidação da Reforma Sanitária Brasileira, com a defesa dos princípios e diretrizes do sistema ${ }^{3}$. É atribuído aos municípios, como responsabilidade na gestão do SUS, a integralidade da atenção à saúde de sua população, garantindo o cumprimento desse princípio por meio de ações prestadas de forma interdisciplinar, com abordagem integral do indivíduo ${ }^{3}$. Assim, devem-se englo- 
bar atividades de promoção da saúde; prevenção de riscos, danos e agravos; e ações de assistência e reabilitação.

Observando essas questões, é possível verificar que a integralidade resume uma das principais e mais marcantes características de nossa política de saúde, já constante no texto de sua Lei Orgânica: a saúde possui uma gama de fatores determinantes e condicionantes 2 . Por tal razão, necessita de uma abordagem pautada em políticas sociais e econômicas e na inter-relação de serviços e ações para a promoção, proteção e recuperação da saúde 2 .

Para Camargo Júnior 4, a palavra integralidade, no contexto brasileiro, não pode ser entendida como um conceito, mas sim como um agrupamento de tendências políticas com relação entre si, o que pode demonstrar tanto fragilidade como potencialidade para o tema. Conill 5 defende que predomina no Brasil a percepção ampliada da integralidade, com ênfase no caráter completo, contínuo e ordenado do cuidado e da gestão. Utilizamo-nos da visão de Mattos 6, ao argumentar que a integralidade é uma "bandeira de luta" e corresponde a um conjunto de valores pelos quais vale lutar, uma vez que se relacionam a um ideal de sociedade mais justa e solidária 6 .

Considerando os diversos enfoques com que é tratada a integralidade, iniciamos com a descrição dos principais grupos de abordagens apresentados pela literatura para o tema da integralidade, em consonância com a classificação estabelecida por Mattos 6:

- Medicina integral: trata das práticas profissionais e milita pela compreensão abrangente das necessidades de saúde dos usuários dos serviços, buscando contemplar necessidades que não se reduzem ao controle das doenças 6 . A luta é contrária à visão reducionista e fragmentada do ser humano, focada nos aspectos biológicos, que reproduz o pensamento cartesiano 7 .

- Integralidade nas políticas específicas: são políticas direcionadas a populações ou portadores de doenças específicas, como idosos e portadores de HIV, com a proposta de abarcar as diversas dimensões envolvidas na questão 6 .

- Integralidade na organização dos serviços: relaciona-se à articulação e coordenação entre os serviços e à garantia da continuidade da assistência 6 . Tal abordagem constitui-se em um desafio ao nosso sistema de saúde, diante do modelo de medicalização, especialização, fragmentação e centrado em hospitais que ainda vivenciamos 7 .

Esta última é a perspectiva que adotamos neste trabalho e se relaciona diretamente com a gestão nos sistemas de saúde. Tal perspectiva é marcada por importantes lacunas na produção de conhecimentos 8 .
Tal dimensão da integralidade trabalha o princípio de forma macro, na articulação de serviços de saúde a uma rede complexa, com outros serviços de saúde e outras instituições, nem sempre necessariamente pertencentes à saúde. Isso é essencial diante do entendimento de que a melhoria das condições de vida é tarefa intersetorial, ainda mais quando se lida com questões de saúde complexas 9 .

Não obstante o fato de que o princípio da integralidade demanda políticas públicas que extrapolam o campo de saúde (como a moradia e o transporte), o exercício prático da integralidade já se apresenta de forma falha quando focamos em ações e serviços específicos da saúde, o que fez emergir a questão deste estudo. Seguindo o pensamento de Machado et al. 10, para que sejam superadas a desintegração e a competição entre órgãos gestores da saúde - que causam ineficiência nas ações -, torna-se indispensável a constituição de redes regionais articuladas.

Isso somente pode ocorrer numa luta cotidiana dentro de serviços, em reuniões de gestores do SUS, nas arenas de negociação e pactuação, ou seja, pautando-se na importância do diálogo 6 , que é o que possibilita a apreensão das necessidades de saúde. Já se entende como ineficiente a atuação de forma separada, como se cada um vivesse em um cenário próprio 11.

Assim, fica evidente a necessidade de se buscar a formação de vínculos entre os órgãos da saúde. Tais vínculos ocorrem em diferentes níveis de complexidade: o primeiro é o do reconhecimento do outro como par, com direito a existir e emitir opiniões; o segundo é o do conhecimento de quem é o outro e como vê o mundo; o terceiro é o da colaboração, com vínculos de reciprocidade; a seguir, está o nível da cooperação, que implica a existência ou a identificação de um problema comum, com uma forma sistemática e estável de atividades; enfim, tem-se o nível de associação com contratos ou acordos e utilização conjunta de recursos 12 .

\section{Vigilância Sanitária e a necessidade de se integrar}

Um grande desafio à aplicação prática da integralidade na saúde é a superação do modelo de atuação centralizador e segregado da Vigilância Sanitária 13. Essa área da saúde pública é definida como espaço de intervenção do Estado com a propriedade de trabalhar no sentido de adequar o sistema produtivo de bens e serviços de interesse sanitário - bem como ambientes - às demandas sociais de saúde e às necessidades do sistema de saúde 14 . 
Atuando sobre fatores de risco associados a produtos e serviços relacionados à saúde, a Vigilância Sanitária se encontra, portanto, no âmbito das relações sociais de produção e consumo, no qual surge grande parte dos problemas de saúde sobre os quais é preciso intervir 15.

É conferida à Vigilância Sanitária, vista como integrante das ações do SUS, uma conceituação abrangente no texto da Lei $n^{\circ} .8 .080$ de 1990: “conjunto de ações capaz de eliminar, diminuir ou prevenir riscos à saúde e de intervir nos problemas sanitários decorrentes do meio ambiente, da produção e circulação de bens e da prestação de serviços de interesse da saúde" 2.

Para Costa 13, tal concepção evidencia, além da esfera mais restrita sobre o risco, que a Vigilância Sanitária possui uma atuação ampla, voltada à garantia do atendimento a necessidades de saúde e resolução de problemas que afetam a integralidade. Na visão de Campos 16, a lei indica a proposta de transformar a Vigilância Sanitária em instrumento de defesa da vida das pessoas.

O Plano Diretor de Vigilância Sanitária (PDVISA), documento que apresenta as diretrizes norteadoras para a consolidação e fortalecimento do Sistema Nacional de Vigilância Sanitária, descreve como prioridade para o sistema um eixo de diretrizes voltado à atenção integral à saúde 17. Ao mesmo tempo, o texto enfatiza a importância da articulação das ações de Vigilância Sanitária com as prioridades das demais políticas públicas 17.

Seguindo essa lógica, focalizamos o presente trabalho no campo da Vigilância Sanitária de serviços de saúde, a qual pretende promover a qualidade da assistência prestada. Para isso, entendese que qualidade do atendimento à população está intrinsecamente relacionada à monitoração dos riscos 18 .

A proteção à saúde da população encontrase, portanto, no cerne da Vigilância Sanitária de serviços de saúde ${ }^{19}$, que trabalha pela prevenção e controle de riscos e eventos adversos relacionados à prestação de serviços de saúde. Para isso, a Vigilância Sanitária de serviços de saúde estabelece práticas direcionadas à melhoria da qualidade da atenção à saúde, seja normalizando os procedimentos, seja adotando medidas (como inspeções e monitoramento) ou fazendo os prestadores cumprirem condições técnicas minimamente necessárias ao funcionamento dos serviços 19. Sua dinâmica de atuação envolve diversos instrumentos e estratégias, sendo a formação de redes de proteção à saúde uma importante ferramenta.
Saúde da Mulher: marco na luta pela integralidade na assistência à saúde

Diversos campos envolvidos com a assistência à saúde possuem discussões e publicações que demandam a integralidade como um fundamento para suas estratégias. Como exemplo, podemos mencionar a saúde mental e a saúde de populações indígenas. Porém uma das temáticas que mais trouxe avanços e propostas à integralidade foi a Saúde da Mulher 6,20. Por muitos anos, o assunto esteve pautado na intervenção sobre os corpos das mulheres-mães, de maneira a assegurar que os corpos dos filhos fossem adequados às necessidades da reprodução social 21.

Por esse motivo e por ser uma área tradicional e consolidada nas secretarias de saúde, elegeu-se a Saúde da Mulher no presente trabalho para representar a assistência à saúde e problematizar sua associação com uma área de relevância da saúde pública: a Vigilância Sanitária. Com isso, há duas temáticas que se apresentam nas instituições públicas, inseridas em dois grandes campos muitas vezes dissociados historicamente em suas práticas: assistência à saúde e saúde pública 10 .

Os movimentos das mulheres retratam batalhas antigas, precedentes ao surgimento do SUS, e que reivindicam reformas das instituições de saúde 22. Como marco histórico, a proposta do Programa de Atenção Integral à Saúde da Mulher (PAISM), na década de 80, demonstrou a necessidade de mudanças na abordagem às mulheres por parte dos serviços, garantindo a visão de sujeito integral e não apenas o foco na assistência ao ciclo gravídico-puerperal.

Embora a imagem do programa tenha se pautado na discussão sobre o princípio da integralidade, o PAISM atuou na perspectiva da resolução de problemas, mantendo a ênfase na saúde reprodutiva e redução da mortalidade materna 23. Encontra-se em vigor, atualmente, a Política Nacional de Atenção Integral à Saúde da Mulher, que apresenta como um de seus princípios a qualidade da assistência e defende, em suas diretrizes, a atenção à mulher nos diferentes níveis do SUS, com articulação das ações e serviços 23.

Corroborando com o que é defendido por Costa, percebe-se que urge a retomada do ideário e da utopia da integralidade na saúde das mulheres, entretanto de forma atualizada e permanente, com as produções científicas que vêm sendo acumuladas nos últimos anos 20.

Muitos dos problemas atuais de Saúde da Mulher estão relacionados à qualidade da assistência 20 , comprovando a urgência em se utilizar e integrar todos os campos do saber da saúde que podem colaborar no enfrentamento desse desafio, entre os quais, está a Vigilância Sanitária. 
É oportuno destacar que, mesmo havendo interfaces importantes nos campos teóricos da Vigilância Sanitária de serviços e a Saúde da Mulher - como a luta pela qualidade dos serviços -, não há publicações que analisem a articulação dessas áreas. Discutir integralidade na Saúde da Mulher não é algo inovador, mas encarar a Vigilância Sanitária como um participante nesse processo é uma abordagem inédita e imperativa, especialmente para os municípios, grandes atores nas novidades e aplicações de políticas públicas.

Considerando esse cenário, tem-se, pois, como objetivo analisar a integração entre a Vigilância Sanitária e a assistência à Saúde da Mulher. Para tanto, parte-se de um estudo de caso, com a seguinte questão norteadora: se e como ocorre a integralidade, na perspectiva da integração, entre a Vigilância Sanitária de serviços de saúde e a área de Saúde da Mulher nas secretarias municipais de saúde?

\section{Metodologia}

Este é um estudo de caso exploratório, de abordagem qualitativa. Os dados foram coletados mediante entrevistas realizadas com 15 profissionais de secretarias de saúde municipais: cinco responsáveis pela Vigilância Sanitária de serviços de saúde (RVS), cinco técnicos da Vigilância Sanitária de serviços de saúde (TVS) e cinco coordenadores de Saúde da Mulher (CSM).

O critério empregado para a seleção dos municípios foi ser capital de estado, uma em cada região brasileira. Isso se justifica pelo fato de as capitais serem pioneiras no processo de descentralização do SUS. Ademais, como se trata de uma pesquisa exploratória, a coleta de dados em distintas regiões do país tornou-se apropriada, de forma a contemplar diferentes realidades locais. Como forma de garantir o sigilo dos sujeitos participantes da pesquisa, não serão abordadas as características das cidades em que ocorreu a pesquisa.

Para os entrevistados, os requisitos foram: manifestarem interesse em participar do estudo após a exposição de informações sobre a pesquisa, serem responsáveis ou técnicos da área de serviços de saúde da Vigilância Sanitária - estes últimos indicados por sua chefia para participarem da pesquisa - ou coordenadores de Saúde da Mulher. Tem-se, portanto, uma amostra de conveniência, vale dizer, que não pretende generalizar os resultados à população, mas que é capaz de desvelar processos importantes à compreensão do assunto.

Durante o planejamento da coleta de dados, foi efetuado contato telefônico com os profissio- nais, como um momento inicial de apresentação da pesquisa, convite para participação e agendamento da viagem da pesquisadora para a realização das entrevistas.

Antes do início das entrevistas, houve explicações sobre o estudo e garantia do anonimato e do sigilo das informações, assim com a assinatura do Termo de Consentimento Livre e Esclarecido. Destaca-se que o estudo foi aprovado pelo Comitê de Ética em Pesquisa da Faculdade de Ciências da Saúde (CEP-FCS) da Universidade de Brasília.

Têm-se como princípio que a entrevista é uma técnica que permite o desenvolvimento de uma estreita relação entre pessoas, configurando-se, pois, em uma comunicação bilateral 24 . Parte, portanto, de uma conduta verbal permeada pelo relato que o entrevistado faz sobre o significado de algo que aconteceu ou está para acontecer 25

Para facilitar a explicitação das percepções dos informantes, utilizou-se roteiro de entrevistas com questões abertas, que foi adotado como um guia para o diálogo. Todas as entrevistas foram gravadas e posteriormente transcritas para a etapa de análise.

Os dados foram trabalhados com o uso da técnica de análise de conteúdo temática 26. O tema é visto como a unidade de registro mais útil em análise de conteúdo 27. Seu uso é comum para pesquisas sobre opiniões, atitudes, valores, crenças, tendências etc. 26 . Pode ser encontrado em uma simples frase, conjunto delas ou em um parágrafo 27 , o que permite que o texto seja recortado em idéias constituintes, enunciados e em proposições com significações isoláveis 26 .

Para a abordagem de cada entrevista, contouse com a participação de três pessoas: o pesquisador principal e dois indivíduos que se propuseram a atuar como juízes na análise do material. A participação de juízes é um mecanismo que proporciona mais validade ao agrupamento de conteúdos em categorias 28.

Cada um dos membros realizou separadamente os seguintes passos em cada entrevista:

- Leitura flutuante da entrevista;

- Novas leituras para demarcação de verbalizações;

- Identificação de temas; e

- Organização dos temas em categorias temáticas que reúnem um grupo de temas, com um título genérico, em razão de caracteres comuns (palavras, expressões e seus significados) 26 .

Com esse trabalho, a equipe se reunia e discutia exaustivamente a análise de cada entrevista, na busca do consenso. Quando não ocorria decidia-se pela definição da maioria. 
Buscou-se, como defendido por Bardin 26, que as categorias fossem uma representação simplificada dos dados brutos. Ao final, com as categorias específicas de cada uma das entrevistas, a equipe avaliava suas co-ocorrências no conjunto de entrevistas e definiam-se as categorias-síntese para cada grupo profissional.

\section{Resultados e discussão}

Os dados sociais dos sujeitos da pesquisa evidenciam alguns pontos relevantes à reflexão. Inicialmente, identificou-se que os CSM possuem mais tempo de trabalho com o tema (18,4 anos, em média, enquanto o tempo foi de 9,4 anos para os RVS e 6,2 anos para os TVS). É pertinente salientar que três desses sujeitos são médicos ginecologistas-obstetras, que desenvolvem atividades relacionadas à Saúde da Mulher desde sua residência médica, o que possivelmente justifica o fato. Também deve se destacar quão diversas são as graduações na formação dos profissionais de Vigilância Sanitária entrevistados (Medicina, Enfermagem, Farmácia, Medicina Veterinária, Nutrição e Odontologia), reiterando a necessidade da interdisciplinaridade nesse campo de saber. No caso dos CSM, a formação acadêmica refere-se aos cursos de Medicina, Enfermagem e Serviço Social.

Para favorecer a visualização dos resultados obtidos com as falas dos sujeitos, estão demonstradas nas Tabelas 1, 2 e 3 as três categoriassíntese de acordo com o grupo de profissionais entrevistados, junto aos temas que as compõem, e exemplos de trechos das entrevistas. Após cada fala, é citado o número da entrevista e a sigla de sua categoria profissional, como mais um mecanismo de codificação e confidencialidade das respostas.

Percebe-se, nos conteúdos obtidos com as entrevistas, que os CSM iniciam o discurso demonstrando o valor da articulação com outras áreas para o desenvolvimento de suas práticas. Ainda que com a organização da secretaria de saúde voltada a grupos populacionais e a patologias específicas, a interação mostra-se fundamental. No caso dos RVS, o trabalho conjunto é compreendido como uma forma de promover qualidade no atendimento ao cidadão.

Apesar do reconhecimento de que a articulação é imprescindível, entende-se, por parte de CSM, que não é fácil de ser aplicada, considerando demandas e questões de poder. Pinheiro 29 afirma que as instituições de saúde representam espaços de luta, de exercícios de poderes, nos

Tabela 1

Categoria síntese dos coordenadores de Saúde da Mulher (CSM).

Título da categoria: "O próprio serviço te obriga a estabelecer essas relações. Mas com a Vigilância Sanitária, não existe relação nenhuma"

$\begin{array}{lll}\text { Temas } & \text { Exemplos de falas }\end{array}$

1 "Não acreditamos em nenhum trabalho isolado"

2 "O próprio serviço te obriga a estabelecer essas relações"

3 "Precisa todo um trabalho em rede"

4 "Não houve nem da parte da mulher nem na parte da Vigilância o enfoque na questão da prevenção"

5 "O trabalho deles (Vigilância Sanitária) é deles. E o nosso é nosso"

6 "A integração dentro de uma secretaria [...] é muito difícil mesmo"

7 A separação física "atrapalha o relacionamento e o crescimento"

8 "Um dos maiores problemas são os gestores"
"Não acreditamos em nenhum trabalho isolado até porque o ser humano é múltiplo" (CSM 2)

"O próprio serviço te obriga a estabelecer essas relações. Por exemplo, na criança tem a questão da imunização. Aí quando você vai trabalhar a Saúde da Mulher, tem a imunização. [...] Então, acaba que a gente, querendo ou não, acaba buscando essa parceria, essa vinculação" (CSM 3)

"Todos os trabalhos deveriam ser em rede, ampliando mais ainda" (CSM 5)

"É uma questão que eu falei no início: se tem um momento de óbito infantil numa UTI ' $x$ ' ou um produto que desconfia que não está de acordo, então levamos o problema pra Vigilância. Mas agora deveria ter uma integração maior na questão da prevenção" (CSM 4)

"O trabalho deles é deles. E o nosso é nosso. Não existe relação nenhuma. Nenhum depende do outro e, quando tem necessidade, por exemplo, às vezes que tenha alguma denúncia, alguma coisa assim, a gente passa pro departamento de controle e avaliação e ele toma as providências" (CSM 3)

"A integração dentro de uma secretaria [...] é muito difícil mesmo. As demandas são muito altas, [...] não tem tempo pra analisar como poderia ter sido feito de uma outra forma, talvez ter feito junto. [...] Acho que tem também as questões de poder" (CSM 1)

"Todos os departamentos são separados. Têm divisórias, é frágil, mas é suficiente para atrapalhar" (CSM 5)

"Eu não tenho dúvida que um dos maiores problemas são os gestores. É a forma como eles se comportam. O modelo que eles reproduzem" (CSM 5) 
Tabela 2

Categoria síntese dos responsáveis pela Vigilância Sanitária de serviços de saúde (RVS).

Título da categoria: "Interface acontece a partir da necessidade"

Temas

Exemplos de falas

1 "Relação com as outras áreas"

"A Vigilância Sanitária vai ter relação com as outras áreas da vigilância em saúde. E com outras instâncias da secretaria de saúde [...]. E a gente tem uma relação também com outras instâncias fora da secretaria da saúde, fora da prefeitura municipal. [...] O Ministério Público que também tem uma relação muito forte com a gente [...]; com o Poder Judiciário" (RVS 1)

2 "Interface acontece a partir da necessidade"

"Infelizmente, essa interface da Vigilância Sanitária com outras áreas da Secretaria de Saúde [...], com a vigilância epidemiológica e controle de avaliação acontece a partir da necessidade. [...] uma agenda pra isso acontecer, infelizmente, eu não consigo perceber" (RVS 3)

3 "Coisas pontuais que a gente procura atender"

4 "Experiência recente" "com a saúde bucal"

5 "Uma articulação com o programa Saúde da Mulher a gente não tem. Talvez não tenha havido problemas"

6 "Não se pensou ainda na possibilidade de trabalhar juntos"

7 “Deveria ter uma proximidade melhor entre a vigilância e a assistência"

8 "Secretaria para um lado e Vigilância para o outro"

9 "Quando a gente trabalha junto, o cidadão ganha"

"Existe [integração] assim, emergencial. [...] Mas não é um programa. São coisas pontuais que a gente procura atender" (RVS 2)

"A gente teve experiência recente e está tendo com a saúde bucal. [...] a gente está fazendo um trabalho junto" (RVS 1)

"Uma articulação com o programa Saúde da Mulher a gente não tem [...] porque talvez não tenha havido problemas de uma maior magnitude nessa área. Se houvesse, eles teriam chamado a Vigilância Sanitária" (RVS 1)

"Eu não percebo esse link. Eu nunca fui chamado pra participar de uma discussão que fechasse essas duas ou que criasse uma proximidade maior entre essas duas esferas" (RVS 3)

"A gente tem uma distância entre a Vigilância Sanitária e a parte da assistência [...]. Eu percebo que deveria ter uma proximidade melhor..." (RVS 2)

"Infelizmente a gente observa que ainda existe uma coisa na secretaria assim: secretaria para um lado e Vigilância para o outro. [...] A própria vigilância epidemiológica, a gente ainda não conseguiu fazer aquele entrosamento que tem tudo a ver. [...] é um desafio essa aproximação" (RVS 5)

"Quando a Vigilância trabalha desarticulada, ela vai lá e vê apenas as questões sanitárias. A gente não verifica a questão de prontuário, de atendimento [...]. E no caso, quando a gente trabalha junto, o cidadão - no caso a mulher - ganha em relação à qualidade. Um atendimento mais amplo, integral e preventivo" (RVS 4) quais os seus atores constroem suas percepções. Dessa forma, a idéia da colaboração se torna um desafio diante dos graus variados de domínio ${ }^{30}$.

Outro tema, também de CSM, remete ao modelo que os gestores propagam. Ferla et al. 31 entendem que a marca centralizadora ainda tem hegemonia nas práticas de gestão de saúde, repercutidas em organizações com pouco diálogo. Outro fator que também dificulta as relações para esse grupo de entrevistados é a separação física: "Mas você fala de Vigilância Sanitária que fica lá... Primeiro que não lembra, depois quando lembra é uma distância assim... Quando você está aqui, você fala: 'ah, preciso falar com fulano'. Ai você corre, vai lá e fala. Então é bem mais ágil. Vigilância Sanitária... Aí marca uma reunião e até que todo mundo consegue... Isso também dificulta um pouco" (CSM 3).

Franco 32 argumenta que há trabalhadores de saúde que, ao desejarem, desenvolvem fluxos de conexões com outros serviços, mesmo diante de um modelo que tenta enquadrá-los em certa conduta para o trabalho. Apostar em processos desse tipo significa se submeter às tensões tão necessárias dos encontros em espaços públicos, na busca de novos sentidos para si e para seu trabalho 33 .

São descritas, por profissionais de Vigilância Sanitária, diversas instituições com as quais possuem relação, destacando-se a vigilância epidemiológica, saúde do trabalhador e auditoria. É abordado por TVS que a integração não faz parte da cultura do trabalho em Vigilância Sanitária, problemática essa abordada no PDVISA, em seu eixo III, ao trazer como diretriz " a articulação permanente das ações de Vigilância Sanitária e os demais serviços e ações de saúde desenvolvidos no âmbito do SUS, garantindo a transversalidade nos diversos níveis de atenção à saúde" 17 (p. 19). 
Tabela 3

Categoria síntese dos técnicos de Vigilância Sanitária de serviços de saúde (TVS).

\begin{tabular}{|c|c|c|}
\hline \multicolumn{3}{|r|}{ Título da categoria: "A gente ainda não divide muito com outros setores" } \\
\hline & nas & Exemplos de falas \\
\hline 1 & $\begin{array}{l}\text { "A gente ainda não divide muito } \\
\text { com outros setores" }\end{array}$ & $\begin{array}{l}\text { "Eu acho que a gente ainda não divide muito com outros setores. [...] A dificuldade maior é a } \\
\text { nossa cultura em cima do 'cada um fazer pelo seu umbigo"" (TVS 4) }\end{array}$ \\
\hline 2 & $\begin{array}{l}\text { "A gente acaba avaliando o risco de } \\
\text { uma forma global e não específica" }\end{array}$ & $\begin{array}{c}\text { "Quando setoriza por Saúde da Mulher especificamente, pra mim fica um pouco mais difícil de perceber a } \\
\text { ação da Vigilância Sanitária [...]. E na verdade a gente acaba avaliando o risco de uma forma } \\
\text { global e não específico, para grupos" (TVS 1) }\end{array}$ \\
\hline 3 & "Necessidades que vão surgindo" & $\begin{array}{l}\text { "A Vigilância Sanitária sempre busca a parceria. De acordo com as necessidades que vão surgindo, [...] } \\
\text { nós estamos presentes com o mesmo objetivo de sempre tentar prevenir os riscos" (TVS 2) }\end{array}$ \\
\hline 4 & $\begin{array}{l}\text { "A partir do momento que fazemos } \\
\text { essas inspeções nós estamos } \\
\text { colaborando" }\end{array}$ & $\begin{array}{c}\text { "A partir do momento que fazemos essas inspeções, nós estamos colaborando com o } \\
\text { programa da Saúde da Mulher" (TVS 2) }\end{array}$ \\
\hline 5 & $\begin{array}{l}\text { "A Vigilância sempre tenta fazer } \\
\text { reuniões explicativas" }\end{array}$ & $\begin{array}{l}\text { "A Vigilância sempre tenta fazer reuniões explicativas. [...] Por exemplo, nós estamos tendo agora um } \\
\text { problema com a parte de pearcing e tatuagens. Tem feito reuniões, tanto com as pessoas que são os } \\
\text { líderes do setor, quanto com o resto da equipe da Vigilância e secretaria de saúde" (TVS 3) }\end{array}$ \\
\hline 6 & $\begin{array}{l}\text { "Tem uma articulação um pouco } \\
\text { maior com a saúde do trabalhador" }\end{array}$ & $\begin{array}{c}\text { "No grupo que eu trabalho a gente inspeciona o ambulatório médico. Aí tem uma articulação um pouco } \\
\text { maior com a saúde do trabalhador" (TVS 1) }\end{array}$ \\
\hline 7 & $\begin{array}{l}\text { "Trabalho conjunto com auditoria } \\
\text { e com o serviço de assistência de } \\
\text { saúde mental" }\end{array}$ & $\begin{array}{c}\text { "A gente faz um trabalho conjunto com auditoria e com o serviço de assistência de saúde mental. [...] } \\
\text { Fazemos um relatório único, então fecha bem a questão sanitária com a assistência, } \\
\text { mais a questão da auditoria" (TVS 4) }\end{array}$ \\
\hline 8 & $\begin{array}{l}\text { "Seria muito interessante repensar } \\
\text { isso pra Saúde da Mulher" }\end{array}$ & $\begin{array}{c}\text { "A gente tem um trabalho muito bom de saúde mental e seria muito interessante repensar isso pra Saúde } \\
\text { da Mulher. Nós fizemos em } 92 \text { um trabalho só com as maternidades. Nós não fizemos em } \\
\text { um conjunto" (TVS 4) }\end{array}$ \\
\hline 9 & $\begin{array}{l}\text { "É com isso que a gente trabalha, o } \\
\text { que vocês podem nos ajudar" }\end{array}$ & $\begin{array}{l}\text { "Eu não sei te falar o que realmente é observado no programa Saúde da Mulher. Talvez se a gente tivesse } \\
\text { uma exposição do que é feito nesse programa, a gente talvez pudesse relacionar o nosso trabalho [...]. } \\
\qquad \text { É uma coisa de aproximar mesmo e dizer: é com isso que a gente trabalha, } \\
\text { o que vocês podem nos ajudar?" (TVS 5) }\end{array}$ \\
\hline
\end{tabular}

É interessante observar que enquanto nos temas de CSM aparece a discussão sobre a integração dentro da secretaria, nos dados dos RVS surge o tema "secretaria para um lado e Vigilância para o outro". Isso evidencia a necessidade de se fortalecer as parcerias da Vigilância Sanitária nas secretarias e encará-la como um componente indissociável do SUS 17.

Emerge de forma relevante nas entrevistas com profissionais de Vigilância Sanitária que as interações acontecem mais por demandas pontuais do que por conta de uma sistematização das relações. O mesmo apareceu como tema na fala dos CSM, ao trazerem a importância de se trabalhar junto com a Vigilância Sanitária em questões de prevenção.

Esse resultado é coerente com o que foi verificado na pesquisa Ações de Vigilância Sanitária na Atenção Básica, que identificou que $87,1 \%$ dos coordenadores do Programa Saúde da Família entrevistados mencionaram que ocorriam articulações com a Vigilância Sanitária por meio do envio de demandas 34 . Também em outro estudo surgiu o fato de que relações da Vigilância Sanitária com outras áreas da vigilância (zoonoses e epidemiologia) só se estabeleciam em casos de surtos ou eventos extraordinários 35 . Paula et al. 30 alertam para essa fragmentação na saúde, representada por relações que se dão apenas por interações pontuais, sem propiciar a troca de saberes.

Os RVS demonstraram preocupação com a distância existente entre a Vigilância Sanitária e a assistência à saúde. Fato semelhante foi citado por Passos 36 para o caso da vigilância epidemiológica: há pouca interação com os programas assistenciais, ressaltando a necessidade de avaliações sobre tais relações em níveis locais.

Nos três grupos profissionais, as falas remetem a pouca ou nenhuma articulação entre a Vigilância Sanitária e a Saúde da Mulher, seja em razão do entendimento de que uma não depende da outra, seja porque nunca se pensou nessa possibilidade de um trabalho conjunto ou porque não houve apresentação de uma área a outra. 
Outro ponto a ser observado é que nos temas dos RVS apareceu o entendimento de que não houve relação, possivelmente pelo fato de não ter havido problemas na coordenação de Saúde da Mulher, pois, caso houvesse, teriam chamado a Vigilância Sanitária. Utilizando-se do Método Paidéia, Campos 16 defende que para o estabelecimento de vínculos, a Vigilância Sanitária deve começar com uma oferta, com um convite à associação, demonstrando suas diretrizes, valores e compromissos. A espera por uma "consulta" ao seu setor é um posicionamento que emperra a construção de redes e favorece o isolamento dos distintos saberes.

Surgiu como tema, por parte de TVS, a percepção de que a realização de inspeções sanitárias seja uma forma de colaboração com a Saúde da Mulher. Essa menção reflete o modelo comumente adotado em saúde: produção de atos 10 que, isoladamente, são ineficazes na resolução de problemas de saúde.

Outro aspecto importante na falas das entrevistas dos técnicos trata-se do tema "a gente acaba avaliando o risco de forma global e não específica", o que é reforçado com o trecho a seguir: "Nós temos no nosso roteiro de inspeção, [...] não de forma específica pra Saúde da Mulher, que écomo te falei, de estar repensando isso de forma geral para toda e qualquer pessoa internada" (TVS 4).

No texto do PDVISA, é ressaltada a necessidade de que a Vigilância Sanitária se articule com ações e programas do SUS destinados a grupos vulneráveis 17 . Isso traz à tona a reflexão sobre quem é o verdadeiro usuário dos serviços prestados pela Vigilância Sanitária. Campos 16 critica a postura comumente adotada de "fechar os olhos" para aqueles que deveriam ser nosso foco, ao afirmar que "a Vigilância Sanitária, a Saúde Pública e a Medicina costumam esquecer-se de que atuam sobre pessoas, valorizando mais as doenças e o ambiente. Estudam as epidemias como se não houvesse sujeitos envolvidos. Intervêm sobre situações de risco como se não mexessem com a vida das pessoas".

O tema dos TVS "a Vigilância sempre tenta fazer reuniões explicativas” traz uma questão que levanta a necessidade de cautela nessas relações. Para que as intervenções na saúde individual e coletiva tenham sucesso, os profissionais devem estar culturalmente sensíveis, contando com informações e participação de todos os recursos humanos e instituições disponíveis 11, fato que vai além de repassar conhecimentos de seu campo de atuação.

A idéia de redes também é pressuposto à efetivação do princípio da integralidade, pensamento demonstrado no tema dos CSM "precisa todo um trabalho em rede". Seguindo essa lógica, experiências exitosas foram lembradas por profissionais de Vigilância Sanitária, como no caso de estratégias desenvolvidas juntamente com a saúde mental e a auditoria: "Primeiro tivemos que definir o que cabia a quem dentro do roteiro de avaliação. O que nós da Vigilância estaríamos nos responsabilizando, quais itens, o que a auditoria estaria se responsabilizando e a saúde mental [...]. É muito rico. De uma forma que não poderíamos fazer isso se não houvesse em conjunto. Um exemplo pra te dar bem prático disso: quando nós fazíamos separados, tinham serviços que eram licenciados e que eram considerados horríveis pela assistência. Um dia eu falei: mas como que esse serviço pra nós é excelência e vocês colocam que está ruim? Então, o documento licença sanitária tem algum problema aí. Ao contrário, também tinha serviço que pra nós era péssimo e pra eles era top de linha de qualidade em assistência à saúde mental. [...] Então a gente tem uma visão do todo que antes a gente não tinha e eles também" (TVS 4).

Esse caso demonstra como é fundamental o entendimento da distribuição de tarefas entre os membros de uma rede, com respeito às competências técnicas, e a necessidade de dispor de momentos para discussão das atividades a serem implementadas, além do reconhecimento das diferenças, possibilitando a identificação de elementos comuns 30 .

Ratifica-se, desse modo, o valor de tecnologias de gestão que promovam condições democráticas de inter-relação dos diferentes conhecimentos para a construção de estratégias ricas e eficazes para o enfrentamento dos problemas prioritários de saúde 22. Experiências desse estilo estimulam e instigam os profissionais a repensarem seu cotidiano.

Os aspectos trazidos pelo estudo constituemse em importantes informações ao entendimento dos obstáculos à efetivação da integralidade, em especial para a Vigilância Sanitária. Torna-se explícita a importância de escutar quem vivencia as negociações políticas e enfrenta as barreiras próprias das organizações de saúde. Os sujeitos da pesquisa confirmam, em suas expressões, o quanto o modelo de atenção do SUS é fragmentado, com permanência do padrão dissociado das ações de assistência e de prevenção.

Nesse contexto, a organização do trabalho nas secretarias de saúde não se permeia pelo princípio da integralidade. Ao contrário, a ausência de planejamento fortalece a dissociação entre as áreas das secretarias. $\mathrm{O}$ foco principal do processo, o usuário do sistema, permanece ignorado em meio às rotinas de cada departamento. Enquanto isso, os principais instrumentos dos quais a secretaria de saúde pode lançar mão para 
planejar suas ações - avaliação de risco e dados epidemiológicos - tornam-se coadjuvantes entre práticas que existem por si mesmas.

Nas falas transparece o isolamento da Vigilância Sanitária e revela-se o quanto é iminente a revisão do método de trabalho da vigilância. Demonstra-se a falta de apropriação das necessidades do campo da atenção à saúde - definidas, no campo federal, pelo Pacto pela Vida - e as sensações de distanciamento em relação à secretaria de saúde. As questões apontadas no Pacto e as diretrizes traçadas no PDVISA são atuais e demandam da Vigilância Sanitária um plano concreto para sua efetivação. Ainda, para reverter essa situação, é fundamental perceber (e fazer) a Vigilância Sanitária não como a “área que realiza inspeção", mas sim como um agente, envolvido na complexidade da saúde, que pode transformar as práticas de assistência à saúde e que se empenha para, junto com os demais, solucionar os problemas do SUS.

Logo, é urgente inserir a Vigilância Sanitária nas mesas de debate, incorporar e trocar conhecimentos a fim de potencializar as ações nas secretarias de saúde. Essa tarefa não é das mais simples, mas somente com o compartilhamento de metas comuns e a reinvenção do trabalho poderemos aplicar a integralidade na realidade do cotidiano. Replicar experiências bem sucedidas, construir e difundir o conhecimento sobre a gestão em redes é tarefa fundamental para que não nos percamos nos discurso da integralidade.

\section{Resumo}

A integralidade é um princípio do SUS, com várias perspectivas, entre as quais a da articulação de seus serviços. O debate sobre esse princípio está presente na assistência à saúde, com destaque para a área de Saúde da Mulher, e nas diretrizes da Vigilância Sanitária. Como as duas áreas buscam a qualidade dos serviços de saúde, o objetivo é analisar a integração entre Vigilância Sanitária e assistência à saúde da mulher. Trata-se de estudo de caso, qualitativo, com entrevistas de profissionais de Vigilância Sanitária de serviços de saúde e coordenadores de Saúde da Mulher (CSM). Os achados apontam para isolamento da Vigilância Sanitária nas secretarias de saúde. A importância da integração é citada por CSM, porém não é fácil de ser aplicada. As relações, se ocorrem, são por situações emergenciais. Os trabalhadores de Vigilância Sanitária acham que não há articulação por não haver problemas na Saúde da Mulher que demandem sua participação e que, com inspeções e palestras, estão colaborando com a área. Mostram-se dificuldades à concretização da integralidade, com o desafio de articular ações em saúde, sobretudo para a Vigilância Sanitária.

Saúde da Mulher; Políticas Públicas de Saúde; Assistência Integral à Saúde; Vigilância Sanitária

\section{Colaboradores}

C. Maia e D. Guilhem foram responsáveis pela concepção do projeto de pesquisa, análise e interpretação dos dados, redação e revisão crítica. G. Lucchese contribuiu com a análise e interpretação dos dados, redação e revisão crítica. 


\section{Referências}

1. National Health System. NHS core principles http://www.nhs.uk/aboutnhs/CorePrinciples/ Pages/NHSCorePrinciples.aspx/ (acessado em 19/ Jul/2009).

2. Brasil. Lei Federal no ${ }^{\circ}$ 8.080/90. Dispõe sobre as condições para a promoção, proteção e recuperação da saúde, a organização e o funcionamento dos serviços correspondentes e dá outras providências. Diário Oficial da União 1990; 19 set.

3. Ministério da Saúde. Diretrizes operacionais dos pactos pela vida, em defesa do SUS e de gestão. Brasília: Ministério da Saúde; 2006.

4. Camargo Júnior KR. As muitas vozes da integralidade. In: Pinheiro R, Mattos RA, organizadores. Os sentidos da integralidade na atenção e no cuidado à saúde. 6a Ed. Rio de Janeiro: Instituto de Medicina Social, Universidade do Estado do Rio de Janeiro/ABRASCO; 2006. p. 13-7.

5. Conill EM. Avaliação da integralidade: conferindo sentido para os pactos na programação de metas dos sistemas municipais de saúde. Cad Saúde Pública 2004; 20:1417-23.

6. Mattos RA. Os sentidos da integralidade: algumas reflexões acerca de valores que merecem ser defendidos. In: Pinheiro R, Mattos RA, organizadores. Os sentidos da integralidade na atenção e no cuidado à saúde. $6 \underline{a}$ Ed. Rio de Janeiro: Instituto de Medicina Social, Universidade do Estado do Rio de Janeiro/ABRASCO; 2006. p. 41-66.

7. Costa-e-Silva V. O processo de implantação do sistema integrado de serviços de saúde em Vitória ES: contribuição à discussão da integralidade na atenção à saúde [Dissertação de Mestrado]. Rio de Janeiro: Escola Nacional de Saúde Pública Sérgio Arouca, Fundação Oswaldo Cruz; 2004.

8. Pinheiro R, Luz MT. Práticas eficazes x modelos ideais: ação e pensamento na construção da integralidade. In: Pinheiro R, Mattos RA, organizadores. Construção da integralidade: cotidiano, saberes e práticas em saúde. 4 a Ed. Rio de Janeiro: Instituto de Medicina Social, Universidade do Estado do Rio de Janeiro/ABRASCO; 2007. p. 9-36.

9. Cecílio LCO. As necessidades de saúde como conceito estruturante na luta pela integralidade e eqüidade na atenção em saúde. In: Pinheiro $R$, Mattos RA, organizadores. Os sentidos da integralidade na atenção e no cuidado à saúde. 6 a Ed. Rio de Janeiro: Instituto de Medicina Social, Universidade do Estado do Rio de Janeiro/ABRASCO; 2006. p. 115-28.

10. Machado MFAS, Monteiro EMLM, Queiroz DT, Vieira NFC, Barroso MGT. Integralidade, formação de saúde, educação em saúde e as propostas do SUS - uma revisão conceitual. Ciênc Saúde Coletiva $2007 ; 12: 335-42$.

11. Meirelles BHS, Erdmann AL. Redes sociais, complexidade, vida e saúde. Ciênc Cuid Saúde 2006; 5:67-74.

12. Feuerwerker LCM, Sousa MF. Em busca de um novo paradigma: a arte de trabalhar em rede. Divulg Saúde Debate 2000; 21:49-53.
13. Costa EA. Vigilância sanitária: proteção e defesa da saúde. In: Rouquayrol MZ, Almeida Filho N. Epidemiologia \& saúde. 6a Ed. Rio de Janeiro: Editora Medsi; 2003. p. 357-87.

14. Lucchese G. Globalização e regulação sanitária: os rumos da vigilância sanitária no Brasil [Tese de Doutorado]. Rio de Janeiro: Escola Nacional de Saúde Pública Sérgio Arouca, Fundação Oswaldo Cruz; 2001.

15. Costa EA, Rozenfeld S. Constituição da vigilância sanitária no Brasil. In: Rozenfeld S, organizadora. Fundamentos da vigilância sanitária. Rio de Janeiro: Editora Fiocruz; 2000. p. 15-40.

16. Campos GWS. Vigilância sanitária: responsabilidade pública na proteção e promoção da saúde. In: Agência Nacional de Vigilância Sanitária. Conferência Nacional de Vigilância Sanitária - caderno de textos. http://www.anvisa.gov.br/divulga/ conavisa/cadernos/eixo1_texto01.pdf (acessado em 17/Set/2007).

17. Agência Nacional de Vigilância Sanitária. PDVISA - Plano Diretor de Vigilância Sanitária: eixos e diretrizes. Brasília: Agência Nacional de Vigilância Sanitária; 2007.

18. Agência Nacional de Vigilância Sanitária. Pediatria: prevenção e controle de infecção hospitalar. Brasília: Ministério da Saúde; 2005.

19. Ministério da Saúde. Direito sanitário e saúde pública. Brasília: Ministério da Saúde; 2003.

20. Costa AM. Atenção integral à saúde da mulher: quo vadis? Uma avaliação da integralidade na atenção à saúde das mulheres no Brasil [Tese de Doutorado]. Brasília: Universidade de Brasília; 2004.

21. Nagahama EEI, Santiago SM. A institucionalização médica do parto no Brasil. Ciênc Saúde Coletiva 2005; 10:651-7.

22. Pinheiro R, Mattos RA. Implicações da integralidade na gestão da saúde. In: Pinheiro R, Mattos RA, organizadores. Gestão em redes: práticas de avaliação, formação e participação na saúde. Rio de Janeiro: Centro de Estudos e Pesquisa em Saúde Coletiva, Instituto de Medicina Social, Universidade do Estado do Rio de Janeiro; 2006. p. 11-26.

23. Ministério da Saúde. Política nacional de atenção integral à saúde da mulher: princípios e diretrizes. Brasília: Ministério da Saúde; 2004.

24. Richardson RJ. Pesquisa social: métodos e técnicas. 3a Ed. São Paulo: Editora Atlas; 1999.

25. Tobar F, Yalour MR. Como fazer teses em saúde pública: conselhos e idéias para formular projetos e redigir teses e informes de pesquisas. Rio de Janeiro: Fundação Oswaldo Cruz; 2001.

26. Bardin L. Análise de conteúdo. 3ạ Ed. Lisboa: Edições 70; 2004.

27. Franco MLPB. Análise de conteúdo. 2a Ed. Brasília: Líber Livro Editora; 2005.

28. Mendonça H, Mendes AM. Experiências de injustiça, sofrimento e retaliação no contexto de uma organização pública do estado de Goiás. Psicol Estud 2005; 10:489-98. 
29. Pinheiro R. As práticas do cotidiano na relação oferta e demanda dos serviços de saúde: um campo de estudo e construção da integralidade. In: Pinheiro R, Mattos RA, organizadores. Os sentidos da integralidade na atenção e no cuidado à saúde. $6 \underline{\text { a }} \mathrm{Ed}$. Rio de Janeiro: Instituto de Medicina Social, Universidade do Estado do Rio de Janeiro/ABRASCO; 2006. p. 67-113.

30. Paula KA, Palha PF, Protti ST. Intersetorialidade: uma vivência prática ou um desafio a ser conquistado? Interface Comum Saúde Educ 2004; 8:331-48.

31. Ferla AA, Ceccim RB, Pelegrini MLM. Atendimento integral: a escuta da gestão estadual do SUS. In: Pinheiro R, Mattos RA, organizadores. Construção da integralidade: cotidiano, saberes e práticas em saúde. 4a Ed. Rio de Janeiro: Instituto de Medicina Social, Universidade do Estado do Rio de Janeiro/ ABRASCO; 2007. p. 63-90.

32. Franco TB. As redes na micropolítica do processo de trabalho em saúde. In: Pinheiro R, Mattos RA, organizadores. Gestão em redes: práticas de avaliação, formação e participação na saúde. Rio de Janeiro: Centro de Estudos e Pesquisa em Saúde Coletiva, Instituto de Medicina Social, Universidade do Estado do Rio de Janeiro; 2006. p. 459-73.
33. Merhy EE. Integralidade: implicações em cheque. In: Pinheiro R, Mattos RA, organizadores. Gestão em redes: práticas de avaliação, formação e participação na saúde. Rio de Janeiro: Centro de Estudos e Pesquisa em Saúde Coletiva, Instituto de Medicina Social, Universidade do Estado do Rio de Janeiro; 2006. p. 97-109.

34. Agência Nacional de Vigilância Sanitária. Ações de vigilância sanitária na atenção básica - relatório de pesquisa. Belo Horizonte: Núcleo de Educação em Saúde Coletiva, Faculdade de Medicina da Universidade Federal de Minas Gerais; 2006

35. Agência Nacional de Vigilância Sanitária. Pesquisa diagnóstico sobre tecnologias em vigilância sanitária. http://www.anvisa.gov.br/servicosaude/ manuais/pesquisa_diagnostico/Volume1_Resul tados.pdf (acessado em 17/Set/2007).

36. Passos LMR. Assistir e vigiar: as ações de vigilância epidemiológica na unidade básica de saúde: situação atual e perspectivas [Tese de Doutorado]. Ribeirão Preto: Universidade de São Paulo; 2003.

Recebido em 27/Jul/2009

Versão final reapresentada em 06/Fev/2010

Aprovado em 17/Mar/2010 